\title{
PROPOSING A LASER BASED BEAM SIZE MONITOR FOR THE FUTURE LINEAR COLLIDER
}

\author{
G.A. Blair ${ }^{a}$, J. Frisch ${ }^{b}$, K. Honkavaara ${ }^{c}$, T. Kamps ${ }^{a *}$, F. Poirier ${ }^{a}$ I. N. Ross ${ }^{d}$, M. Ross ${ }^{b}$ \\ H. Schlarb ${ }^{c}$, P. Schmüser ${ }^{c}$, S. Schreiber ${ }^{c}$, D. Sertore ${ }^{e}$, N. Walker ${ }^{c}$, M. Wendt ${ }^{c}$, K. Wittenburg $^{c}$ \\ ${ }^{a}$ Royal Holloway University of London, Egham, Surrey, TW20 0EX, UK \\ ${ }^{b}$ Stanford Linear Accelerator Center, Stanford, CA 94309, USA \\ ${ }^{c}$ Deutsches Elektron-Synchrotron DESY, D-22603 Hamburg, Germany \\ ${ }^{d}$ Rutherford Appleton Laboratory, Chilton, Didcot, Oxon OX11 0QX, UK \\ ${ }^{e}$ INFN Milano LASA, I-20090 Segrate (MI), Italy
}

\begin{abstract}
Compton scattering techniques for the measurement of the transverse beam size of particle beams at future linear colliders (FLC) are proposed. At several locations of the beam delivery system (BDS) of the FLC, beam spot sizes ranging from several hundreds to a few micrometers have to be measured. This is necessary to verify beam optics, to obtain the transverse beam emittance, and to achieve the highest possible luminosity. The large demagnification of the beam in the BDS and the high beam power puts extreme conditions on any measuring device. With conventional techniques at their operational limit in FLC scenarios, new methods for the detection of the transverse beam size have to be developed. For this laser based techniques are proposed capable of measuring high power beams with sizes in the micrometer range. In this paper general aspects and critical issues of a generic device are outlined and specific solutions proposed. Plans to install a laser wire experiment at an accelerator test facility are presented.
\end{abstract}

\section{MOTIVATION}

High luminosity is the key to many of the physics processes of special interest at the Future Linear Collider [1]. This fundamental point is the main physics motivation for this project and justifies considerable efforts to ensure that the accelerator can deliver on its excellent luminosity potential. The case for the highest luminosities is now globally accepted and all the Linear Collider proposals currently have this as their goal, with quoted luminosities of a few $\times 10^{34} \mathrm{~cm}^{2} \mathrm{~s}^{-1}$. The key motivation for this project is to add to the arsenal of tools that the machine will need to maximize its luminosity performance. In particular this project aims to provide a reliable and flexible method of obtaining real-time information on the emittance and quality of the beam and hence to allow feedback for maximizing the luminosity.

\footnotetext{
*t.kamps@rhul.ac.uk
}

\section{EMITTANCE MEASUREMENT}

In this project we limit our attention to the measurement of the electron beam transverse phase space (transverse emittance) because it is the fundamental determining factor for the final transverse beam-spot size at the interaction point (IP). It is important to keep the emittance low so as to maximize the luminosity at the IP and much effort is spent in designing the accelerator and beam delivery system (BDS) to avoid sources of emittance growth. The BDS generically consists of approximately a kilometer of beam optics providing collimation, chromatic correction and final focusing. There are many potential sources of emittance growth which in general will be time dependent and will require continuous measurement and feedback to correct.

The aim is to measure the emittance of the beam to better than $10 \%$ as it approaches the IP and this will require a number of profile measurements with the same precision along the BDS. In Tab. 1 beam profile parameters for FLC designs are listed.

\begin{tabular}{lcccc} 
& & CLIC & NLC/JLC & TESLA \\
\hline \multirow{2}{*}{ BDS } & $\sigma_{x}[\mu \mathrm{m}]$ & 3.4 to 15 & 7 to 50 & 20 to 150 \\
& $\sigma_{y}[\mu \mathrm{m}]$ & 0.35 to 2.6 & 1 to 5 & 1 to 25 \\
\hline \multirow{2}{*}{ IP } & $\sigma_{x}^{\star}[\mathrm{nm}]$ & 196 & 335 & 535 \\
& $\sigma_{y}^{\star}[\mathrm{nm}]$ & 4.5 & 4.5 & 5 \\
\hline
\end{tabular}

Table 1: Beam spot sizes for various Linear Collider designs. Quoted are numbers for CLIC [2], NLC/JLC [3], and TESLA [4].

A set of transverse profile measurements at several points along the beam line separated by a sufficient betatron phase advance can be translated into a determination of the emittance. At least four scanning stations will be required for each lepton beam, possibly fired by a single laser system plus laser beam transportation. Each station will need to provide a profile along three directions, as required to specify an ellipse. Relating a set of such transverse profiles to the emittance and optimizing the layout of scanning stations within a BDS design will form an interesting parallel 
project, that will be addressed via detailed simulations.

The electron bunch transverse profile has been measured in the past by intersecting the electron beam with a solid wire and by counting the subsequent background rate as a function of the relative position of wire and bunch. Using this technique, resolutions of typically a few $\mu \mathrm{m}$ can be obtained, at the expense of some disruption to the beam. This technique cannot be used universally at the LC, however, because the beam-spot sizes can be much smaller, the need for continuous measurement precludes an invasive technique and the intensities are so great that the wires would be quickly damaged, even if swept rapidly through the beam. For these reasons, it is necessary to develop a novel technique that can run continuously and reliably during machine operation, that does not get destroyed by the beam and that can be sufficiently fast so as to be sensitive to individual electron bunches within the bunch train. All these advantages could in principle be provided using optical scattering structures.

\section{COMPTON SCATTERING}

The basic idea is to replace a solid wire in a beam profile scan by a narrow beam of laser light. The fundamental process at work is then the Compton effect, where photons are scattered out of the laser beam by the incoming electrons. By counting the rate of scattered photons (or electrons) as a function of the relative position of the laser waist and the electron beam, a measurement of the bunch transverse profile can be obtained. The relativistic Compton process has been studied in detail elsewhere [5]. Relevant aspects for our analysis together with detailed simulations are collected in [6].

Several schemes have been proposed to use optical scattering structures to serve as diagnostics to measure the bunch length and the beam profile [7].

\subsection{OPTICAL SCATTERING STRUCTURES}

Common to all optical scattering structures is that they must have features smaller or similar in size to the particle beam under measurement. Several types of laser spot structures can be generated with common optical setups. In the following some optical structures are listed together with their performance rating:

Laser wire (gaussian profile) The laser beam is here focused to a small gaussian spot with radius $\omega_{o}$. If we consider a diffraction limited, finely focused beam waist, the minimal achievable spot radius is given by $\omega_{o}=\lambda /(\pi \theta)$, where $\lambda$ denotes the laser wavelength and $\theta$ the half opening angle of the laserbeam at the waist (see Fig.1). The distance over which the laser beam diverges to $\sqrt{2}$ of its minimum size is called the Rayleigh range $x_{R}$ and defines the usable length of the laser wire. The smallest achievable spot size with diffraction limited optics is in the order of $\omega_{o} \sim \lambda$. With Nd:YLF or YAG laser working at higher harmonics electron spot sizes from $\sigma_{y}>350 \mathrm{~nm}$
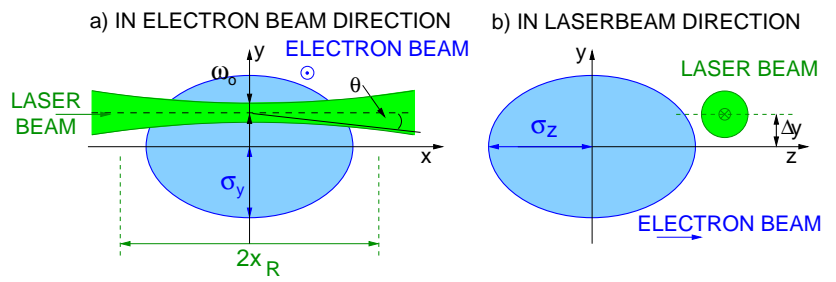

Figure 1: Scheme of a gaussian laser beam focused to its diffraction limit scanned over an electron beam.

can be measured with high accuracy. The laser beam power must be in the order of a couple of MW to yield a few thousand Compton photons per scan spot. Critical issues of a laser wire design are the diffraction limited optics, which must withstand such a high beam power and the scanning system, enabling intra-train scanning of consecutive bunches.

Laser wire (dipole mode) The resolution of the laser wire can be enhanced by generating an artificial transverse dipole mode by means of a lambda half waveplate, where half of the gaussian is shifted in phase by $90^{\circ}$. Such a waveplate can easily be installed in the optical path of the laser wire and would enhance the resolution of the device by roughly a factor of two aiming at beam sizes in a region from $250 \mathrm{~nm}<\sigma_{y}<500 \mathrm{~nm}$.

Laser Interferometer Towards beam sizes in the nanometer range, a standing wave interference pattern generated by crossing two laser beams has been proposed and successfully tested at the FFTB experiment [8]. The fringe spac-

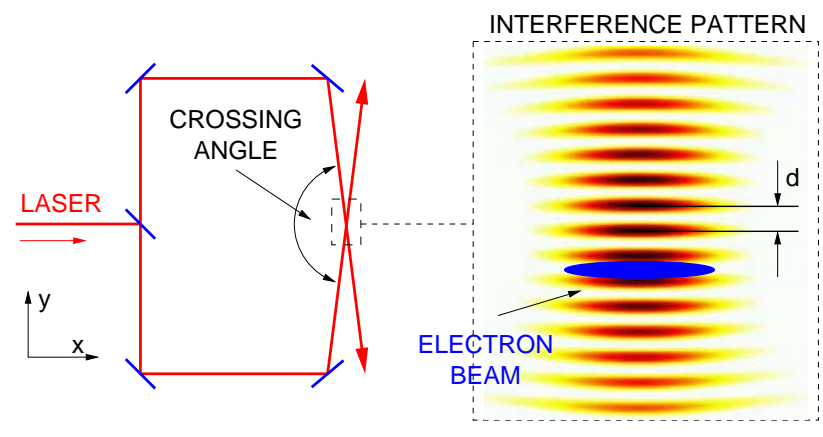

Figure 2: Scheme for the generation of an interference pattern with fringe spacing $d$.

ing of the interference pattern (see Fig. 2) depends on the laser wavelength and on the crossing angle. The electron beam is moved over the pattern and the Compton scattered photons are detected. If the beam size is small compared to the fringe spacing, a modulation of the Compton signal is observed which is proportional to the transverse electron beam size. This modulation vanishes if the beam size is large compared to the fringe spacing. The smallest observed spot size with this technique was about $58 \mathrm{~nm}$ [9]. 


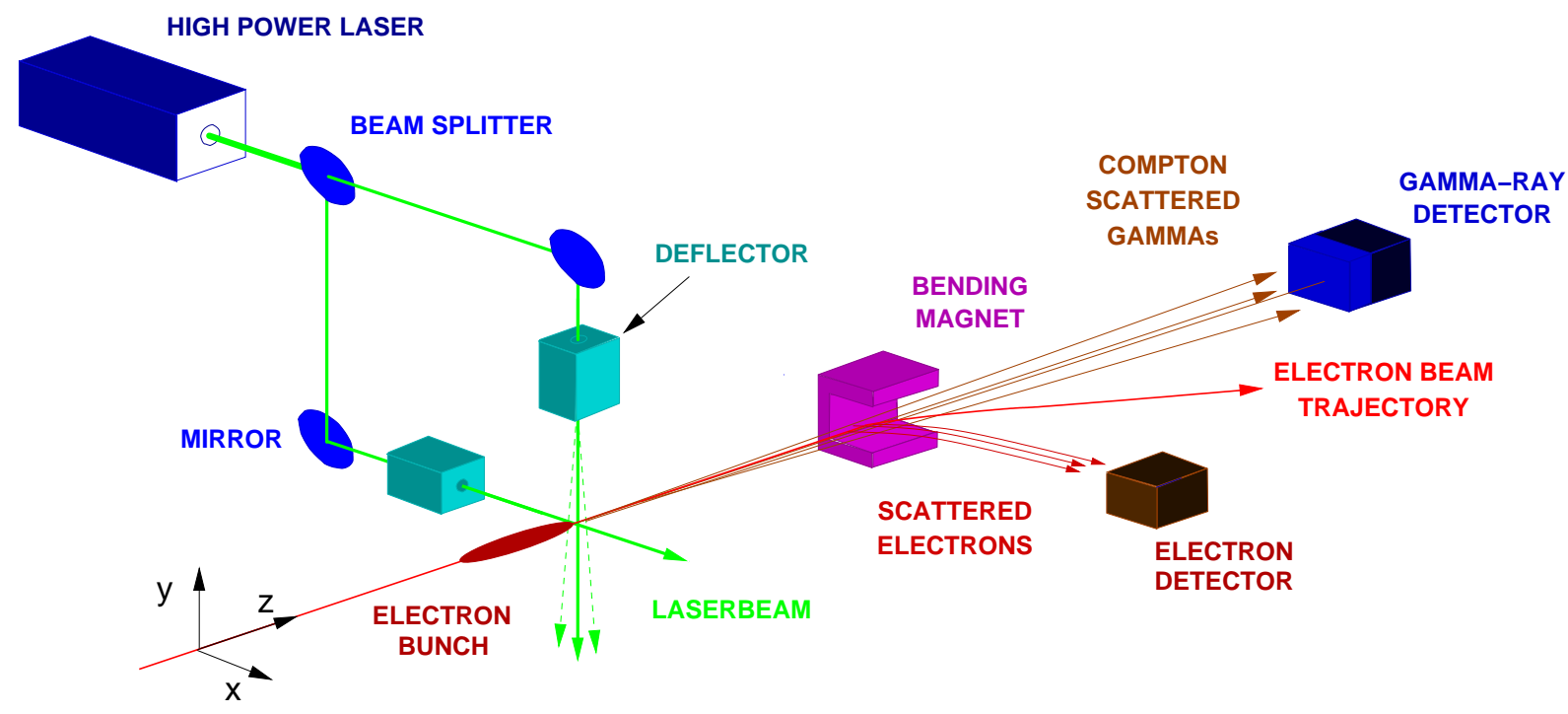

Figure 3: Schematic setup for a laser wire beam profile monitor.

\section{LASER WIRE SETUP}

The setup for a laser wire beam profile monitor is sketched in Fig. 3. A high power laser beam is divided into two different optical paths for scanning the horizontal and vertical beam size. The scanning is foreseen to be done either with piezo-driven mirrors or with acousto-optic scanners. Before the interaction with the electron beam the laser beams are focused. The electron beam is then bent away while the Compton scattered photons travel along a straight line where they are detected with a calorimeter. Scattered electrons will be bent more strongly than particles with the nominal beam energy enabling detection at a location after the bending magnet.

\section{TEST OPTIONS}

It is planned to install a complete laser wire scanner at the PETRA accelerator at DESY in summer 2002. While beam sizes at PETRA $(10-100 \mu \mathrm{m})$ are comparable with typical FLC BDS numbers, the energy of the electron beam is lower in the range from $4.5-12 \mathrm{GeV}$. Recent results from background measurements show [6] that a sufficient signal to noise ratio can be reached even with a medium power laser with peak power less than $10 \mathrm{MW}$. In Spring 2002 tests of subsystems of a laser wire are planned at CTF2/3.

\section{CONCLUSIONS}

It is anticipated that laser wire scanners will be the standard beam size instrumentation tool for the beam delivery system of all FLC designs. First design ideas exist with the prototype setup tested at SLC/SLD [10]. Our aim is to elevate this design to a compact, non-invasive device where a high-power pulsed laser is scanned across the electron beam with novel scanning techniques.

\section{REFERENCES}

[1] The various worldwide Linear Collider workshop proceedings and documentation of ongoing activities can be accessed from the central website: http://lcwws.physics . yale.edu/lc/

[2] R. Assmann et al., "Design status of the CLIC 3-TeV beam delivery system and damping rings," CERN-SL-2000-058AP.

[3] P. Tenenbaum, L. Eriksson, T. Markiewicz, T. O. Raubenheimer and A. Ringwall, "New developments in the Next Linear Collider beam delivery system design," SLAC-PUB8135 Contributed to IEEE Particle Accelerator Conference (PAC 99), New York, NY, 29 Mar - 2 Apr 1999.

[4] R. Brinkmann, N. Walker, O. Napoly and J. Payet, "A new beam delivery system (BDS) for the TESLA linear collider," DAPNIA-SEA-00-04.

[5] I. F. Ginzburg, G. L. Kotkin, V. G. Serbo and V. I. Telnov, "Colliding Gamma E And Gamma Gamma Beams From Single Pass Electron Positron Accelerators," Sov. J. Nucl. Phys. 38 (1983) 222

[6] K. Balewski, G. A. Blair, T. Kamps, F. Poirier, D. Sertore, K. Wittenburg, "Simulation Studies and Background Measurements for a Laser Based Beam Size Monitor for Future Linear Collider," PAC 2001, Chicago, TPAH052, this proceedings

[7] T. Shintake, "Proposal of Nanometer beam size monitor for e+ e- linear colliders,” Nucl. Instrum. Meth. A 311 (1992) 453.

[8] V. Balakin et al., "Focusing of submicron beams for $\mathrm{TeV}$ Scale e+ e- linear colliders," Phys. Rev. Lett. 74 (1995) 2479.

[9] P. Tenenbaum and T. Shintake, "Measurement of small electron-beam spots," Ann. Rev. Nucl. Part. Sci. 49 (1999) 125.

[10] R. Alley et al., "A laser-based beam profile monitor for the SLC/SLD interaction region," Nucl. Instrum. Meth. A 379 (1996) 363. 